\title{
Urgent Necessity for Standardized and Evidence Based Plant Immunomodulators (Such As Rice Bran Arabinoxylan Concentrate/MGN-3) for the Tumor Research
}

\begin{abstract}
Biological targeting therapies can inhibit the cascade of cell proliferation and enhance the sensitivity of malignant tumor cells against natural immune effector cells. Clinical observations suggest that their combination with evidence based and standardized plant immunomodulators (such as arabinoxylan concentrate using Biobran/MGN-3) can induce astonishing results.

Since the escape of tumor cells from $\mathrm{T}$ lymphocytes is well known, growing interest is focusing on the Pathogenic Associated Molecular Pattern (PAMP) molecules which are able to stimulate the so called type- 1 natural antitumor mechanisms in a MHC unrestricted manner. However, PAMP molecules exist only in the nature (bacteria and plants). The chemistry is not able to produce them. Bacteria are toxic. Therefore growing interest developed for the PAMP like molecules in the plants. Unfortunately, in terms of PAMP like molecules standardized plant immunomodulators (such as arabinoxylan concentrate in Biobran/MGN-3) are registered world over as food supplement and therefore their further clinical research in various oncological centers is inhibited.
\end{abstract}

Keywords: MEK-inhibitor; Immunomodulatory treatment; Tumor disease; Rice bran arabinoxylan; MGN-3

Review Article
Volume 9 Issue 3 - 2017
Tibor Hajto*
Institute of Pharmaceutical Chemistry, Medical University
Pecs, Hungary
*Corresponding author: Hajto T, Institute of
Pharmaceutical Chemistry, Medical University Pecs, Meggyes
u. 26, H-2045, Törökbálint, Hungary, Tel: +36 309735 337;
Email: drhajtot@t-online.hu
Received: October 31, 2017 | Published: November 10,
2017

\section{Introduction}

In the last time a case report was published that a MEK (Mitogen activated Extracellular signal regulation Kinase) inhibitors which can down regulate the receptor tyrosine kinase mediated signaling pathway [1-3] given in a combination with a standardized and evidence based plant immunomodulators was able to induce complete remissions of lung, liver and brain metastases [4]. Since these therapy modalities can't result in similar astonishing clinical responses if they are applied separately [5] the hypothesis arouse that without standardized and evidence based plant immunomodulators the further tumor research is impeded.

In the last decades the immunology exhibited an enormous progress. However, the clinical research of malignant tumors for a long time was not able to profit too much from this development. In the last years, new biological therapeutic efforts with targeting strategy opened new perspectives for the tumour immunology. Growing evidences suggest that inhibition of Growth Factor signaling or blocking several mechanisms can also improve the tumour-induced disturbance of the immune regulation. Despite the improved outcomes with monoclonal antibodies-related targeting therapies, durable responses are uncommon [5]. Recently, it was also found that these new biological therapeutic efforts with targeting strategy can enhance the expression of stress related ligands (MICA, MICB , ULBP1 etc.) on tumour cells resulting in their increased sensitivity to natural effectors [6-7]. There is now a logical conclusion that the monoclonal targeting therapy and natural effectors activating immunomodulatory treatment together may open new perspectives in the tumor research.

Pathogenic Associated Molecular Pattern (PAMP) Molecules in Plants are Promising Therapeutic Tools in the Treatment of Malignant Tumors

Since more than 100 years it was repeatedly observed that bacteria are able to improve the immune defense against tumors. Today, it is well known that the so called Pathogenic Associated Molecular Pattern (PAMP) molecules are responsible for this beneficial effect. As it is also well known, PAMP molecules exist on membrane of bacteria which are able to bind Pattern Recognition Receptors (PRR) on phagocytic cells inducing an activation of the cascade of effector cells which are potent inhibitors of tumor growth. However, the use of bacteria or their active components in tumor treatment was always very limited since they are toxic and the attempts to diminish their toxicity caused damages in the structure of their PAMP molecules. Namely, always more data support that not the chemical composition but the configuration of PAMP molecules is important for their biological 
effects. Consequently, the chemistry is not able to produce PAMP configurations but fortunately plants have also PAMP like molecules which are mostly not toxic substances. We need in term of PAMP like molecules standardized, immunological and clinical evidence based plant immunomodulators. The first plant immunomodulator which can correspond to these requirements is a standardized Rice Bran Arabinoxylan Concentrate (BioBran/ MGN-3) which also as food supplement is registered in spite of the immunological and clinical (based on randomized double blind trial) evidences [8-22]. Unfortunately, oncological centers are not ready to carry out further investigations with food supplements and arabinoxylan concentrate can't take part on further controlled clinical trials. Therefore we must create a social network for the acceptance of evidence based immunomodulatory therapy with plant origin.

The tumor research needs urgently effective immunomodulators since in the least years new perspectives are opened by targeting of important steps in biological regulation. Growing evidence suggests that a similar polarity exists in the innate immunity and in the neuroendocrine system. However, until now the role of their disturbed regulation observed in the tumour disease was only by the immunological research discussed. Indeed, available information suggests that tumorassociated (type-2) immune cells affect chronic inflammation, promote cell proliferation by producing growth factors, stimulate angiogenesis and inhibit the so called type- 1 cells which are potent inhibitors of tumor growth [23-24]. In this tumor-induced disturbance of natural immune system the neuroendocrine system takes also part. Therefore the research of the relationship between immune and neuroendocrine regulation will be also very exciting and promising.

\section{Conclusion}

The evidence based and standardized plant immunemodulators must be more available for the tumor research since their combination with the new biological targeting therapy modalities may open new perspectives in the tumor treatment.

\section{Acknowledgment}

None.

\section{Disclosure Statement}

The author declares that there is no competing or other conflicting interest in relation to this paper.

\section{References}

1. Li L, Zhao GD, Shi Z, Qi LL, Zhou LY, et al. (2016) The Ras/Raf/ MEK/ERK signaling pathway and its role in the occurrence and development of HCC. Oncol Lett 12(5): 3045-3050.

2. Chaparro M, González Moreno L, Trapero-Marugán M, Medina J, Moreno-Otero R (2008) Review article: pharmacological therapy for hepatocellular carcinoma with sorafenib and other oral agents. Aliment Pharmacol Ther 28(11-12): 1269-1277.

3. Stinchcombe TE, Johnson GL (2014) MEK inhibition in non-small cell lung cancer. Lung Cancer 86(2): 121-125.
4. Hajto T (2017) Can standardized plant immunomodulator (rice bran arabinoxylan concentrate/MGN-3) increase the effects of MEK and BRAF inhibitors with clinical benefit? Case report of a patient with carcinoma in biliary duct. Res Rew Insight 1(3): 1-4.

5. Smith MP, Wellbrock C (2016) Molecular Pathways: Maintaining MAPK Inhibitor Sensitivity by Targeting Nonmutational Tolerance. Clin Cancer Res 22(24): 5966-5970.

6. Bae JH, Kim SJ, Kim MJ, Oh SO, Chung JS, et al. (2012) Sussceptibility to natural killer cell-mediated lysis of colon cancer cells is enhanced by treatment with epidermal growth factor receptor inhibitors through UL16 binding protein-1 induction. Cancer Sci 103: 7-16.

7. He S, Yin T, Li D, Gao X, Wan Y, et al. (2013) Enhanced interaction between natural killer cells and lung cancer cells: involvement in gefitinib-mediated immunoregulation. J Transl Med 11: 186.

8. Cholujova D, Jakubikova J, Czako B, Martisova M, Hunakova L, et al. (2013) MGN-3 arabinoxylan rice bran modulates innate immunity in multiple myeloma patients. Cancer Immunol Immunother 62(3): 437-445.

9. Cholujova D, Jakubikova J, Sedlak J (2009) BioBran-augmented maturation of human monocyte-derived dendritic cells. Neoplasma 56(2): 89-95.

10. Tan BL, Norhaizan ME (2017) Scientific Evidence of Rice ByProducts for Cancer Prevention: Chemopreventive Properties of Waste Products from Rice Milling on Carcinogenesis In Vitro and In Vivo. Biomed Res Int 2017: 9017902.

11. Pérez-Martínez A, Valentín J, Fernández L, Hernández-Jiménez E, López-Collazo E, et al. (2015) Arabinoxylan rice bran (MGN-3/ Biobran) enhances natural killer cell-mediated cytotoxicity against neuroblastoma in vitro and in vivo. Cytotherapy 17(5): 601-612.

12. Badr El-Din NK, Ali DA, Alaa El-Dein M, Ghoneum M (2016) Enhancing the Apoptotic Effect of a Low Dose of Paclitaxel on Tumor Cells in Mice by Arabinoxylan Rice Bran (MGN-3/Biobran). Nutr Cancer 68(6): 1010-1020.

13. Badr El-Din NK, Abdel Fattah SM, Pan D, Tolentino L, Ghoneum M (2016) Chemopreventive Activity of MGN-3/Biobran Against Chemical Induction of Glandular Stomach Carcinogenesis in Rats and Its Apoptotic Effect in Gastric Cancer Cells. Integr Cancer Ther 15(4): NP26-NP34.

14. Ghoneum M, Agrawal S (2014) Mgn-3/biobran enhances generation of cytotoxic CD8+ T cells via upregulation of dec-205 expression on dendritic cells. Int J Immunopathol Pharmacol 27(4): 523-530.

15. Ghoneum M, Badr El-Din NK, Ali DA, El-Dein MA (2003) Modified arabinoxylan from rice bran, $\mathrm{MGN}-3$ /biobran, sensitizes metastatic breast cancer cells to paclitaxel in vitro. Anticancer Res 34(1): 8187.

16. Ghoneum M, Badr El-Din NK, Abdel Fattah SM, Tolentino L (2013) Arabinoxylan rice bran (MGN-3/Biobran) provides protection against whole-body $\gamma$-irradiation in mice via restoration of hematopoietic tissues. J Radiat Res 54(3): 419-429.

17. Ghoneum M, Agrawal S (2011) Activation of human monocytederived dendritic cells in vitro by the biological response modifier arabinoxylan rice bran (MGN-3/Biobran). Int J Immunopathol Pharmacol 24(4): 941-948.

18. Badr El-Din NK, Noaman E, Ghoneum M (2008) In vivo tumor inhibitory effects of nutritional rice bran supplement MGN-3/ 
Biobran on Ehrlich carcinoma-bearing mice. Nutr Cancer 60(2): 235-244.

19. Gollapudi S, Ghoneum M (2008) MGN-3/Biobran, modified arabinoxylan from rice bran, sensitizes human breast cancer cells to chemotherapeutic agent, daunorubicin. Cancer Detect Prev 32(1): 1-6.

20. Bang MH, Van Riep T, Thinh NT, Song le H, Dung TT, et al (2010) Arabinoxylan rice bran (MGN-3) enhances the effect of interventional therapies for the treatment of hepatocellular carcinoma: A three-year rabdomized clinical trial. Anicancer Res 30(12): 5145-5152.

21. Itoh Y, Mizuno M, Ikeda M, Nakahara R, Kubota S, et al. (2015) A Randomized, Double-Blind Pilot Trial of Hydrolyzed Rice Bran versus Placebo for Radioprotective Effect on Acute Gastroenteritis Secondary to Chemoradiotherapy in Patients with Cervical Cancer. Evid Based Complement Alternat Med 2015: 974390.

22. McDermott C, Richards SC, Thomas PW, Montgomery J, Lewith G (2006) A placebo-controlled, double-blind, randomized controlled trial of a natural killer cell stimulant (BioBran MGN-3) in chronic fatigue syndrome. QJM 99(7): 461-468.

23. Mantovani A (2007) Inflammation and cancer; the macrophage connection. Medicina (Buenos Aires) 67(Suppl II): 32-34

24. Sanches-Torres C, Garcia-Romo GS, Cornejo-Cortes MA et al. (2001) CD16+ and CD16- human blood monocyte subsets differentiate in vitro to dentritic cells with different abilities to stimulate CD4+ T cells. Int Immunol 13(12): 1571-1581. 